\title{
Physical inactivity and television-viewing time among Aboriginal adults with asthma: a cross-sectional analysis of the Aboriginal Peoples Survey
}

\author{
N. Doggett, BKin (1); S. Dogra, PhD (2)
}

This article has been peer reviewed.

Tweet this article

\section{Abstract}

Introduction: The purpose of this analysis was to 1) determine the association between asthma and physical activity levels or sedentary time among Aboriginal adults, and 2) understand the influence of physical inactivity and sedentary time on health care use among Aboriginal adults with asthma.

Methods: We analyzed 20953 adults from the 2006 Aboriginal Peoples Survey. Those with self-reported physician-diagnosed asthma and a current prescription for asthma medication were considered to have current asthma. Insufficient physical activity was defined as $<3$ hours/week of moderate to vigorous physical activity; high television screen time was defined as > 10 hours/week. Health care use was assessed using the number of health professional visits and overnight hospital stays.

Results: Aboriginal adults with asthma were more likely to report high televisionviewing time $(\mathrm{OR}=1.16$; $\mathrm{CI}: 1.11-1.22)$ and insufficient physical activity $(\mathrm{OR}=1.15$; CI: 1.10-1.20) than those without asthma. Those with asthma who reported high television-viewing time reported more health professional consults in the past 12 months $(\mathrm{OR}=2.59$; CI: 2.34-2.87), more overnight stays in hospital in the past year $(\mathrm{OR}=1.95$; CI: $1.82-2.08)$ and more overnight stays in the hospital in the past 5 years $(\mathrm{OR}=1.13$; CI: 1.07-1.18); results were less consistent for physical activity and health care use.

Conclusion: These findings suggest that Aboriginal adults with asthma are less active than their peers without asthma and that such a lifestyle may be associated with higher health care use. These findings have implications for physical activity promotion and sedentary behaviour strategies targeting Aboriginal adults with asthma.

Keywords: physical activity, sedentary lifestyle, chronic disease, asthma control

\section{Introduction}

Aboriginal adults in Canada have a disproportionately high burden of chronic disease, increased mortality and decreased life expectancy. ${ }^{1}$ Research has shown that off-reserve First Nations, Métis and Inuit adults also have a higher prevalence of asthma $(13 \%-14 \%)$ compared to nonAboriginal adults (8.6\%). ${ }^{2}$ Low socioeconomic status, limited access to health care services and poor treatment adherence are partly responsible for this discrepancy. ${ }^{3}$

Between 1998 and 2001, asthma-related symptoms were responsible for more than 80000 hospital admissions. ${ }^{4}$ In 2011, the overall economic burden of asthma in Canada, including direct costs and indirect costs, was more than $\$ 2$ billion. ${ }^{5}$ Similar data on asthma-related hospitalizations among Aboriginal people is not available.
Key findings

- Aboriginal adults with asthma are more sedentary and less physically active than their peers without asthma.

- Further, Aboriginal adults with asthma who report high levels of television-viewing time are more likely to use health care services than their less sedentary peers.

However, a longitudinal study in Saskatchewan found that Registered Indians aged 35 to 64 years showed a significantly higher risk of hospitalization for asthma. ${ }^{6}$ Another retrospective cohort study in Alberta found that Treaty Indians were 2 times more likely to visit a hospital emergency department for asthma or COPD-related symptoms than non-Aboriginal people; however, they were less likely to see a specialist or undergo spirometry testing. ${ }^{3}$

Research has shown that physical activity reduces the incidence and may help prevent the progression of conditions such as diabetes, high blood pressure, cardiovascular disease, asthma, arthritis and poor health. ${ }^{7,8}$ The most recent data on physical activity rates among Aboriginal people in Canada indicate that only $21 \%$ of on-reserve First Nations are physically active ${ }^{9}$ compared to $53.8 \%$ of non-Aboriginal people. ${ }^{10}$ Lower physical activity rates may partially explain the higher incidence and worse management of chronic disease in this population. ${ }^{9}$ Regular physical activity is associated with improved asthma control ${ }^{11}$ as well as lower health care use. ${ }^{12}$ Activity limitation is an important criteria in the assessment of asthma control. ${ }^{13}$

\section{Author references:}

1. School of Recreational Management and Kinesiology, Acadia University, Wolfville, Nova Scotia, Canada

2. Faculty of Health Sciences, Kinesiology, University of Ontario Institute of Technology, Oshawa, Ontario, Canada

Correspondence: Shilpa Dogra, Assistant Professor, University of Ontario IT, 2000 Simcoe St N, Oshawa, ON L1H 7K4; Tel: 905-721-8668 ext. 6240; Email: shilpa.dogra@uoit.ca 
Unfortunately, there is a dearth of data on the effect of sedentary behaviour on asthma control. Recent research indicates that sedentary behaviour, which refers to time spent sitting while commuting, working or during leisure time, ${ }^{14}$ is an independent risk factor for chronic morbidity and all-cause mortality. ${ }^{15}$ A large proportion of leisure sedentary time is made up of screen time, specifically, television time. ${ }^{16}$ In fact, television time contributes to the highest amount of daily screen time among Canadian adults, with $29 \%$ of this population reporting 15 or more hours a week ( $>2$ hours per day) in $2007 .{ }^{17} \mathrm{~A}$ recent study also found that $64 \%$ of Metis reported watching $\mathrm{TV}$ for more than 6 hours per week. ${ }^{18}$ However, the impact of such sedentary activities on asthma outcomes is unclear.

Aboriginal people in Canada have a higher prevalence of asthma ${ }^{2}$, appear to have worse asthma control ${ }^{19}$ and have higher levels of physical inactivity. ${ }^{9}$ Given the established link between physical activity and asthma-related health outcomes, a better understanding of the association between asthma, engaging in regular physical activity and having high levels of sedentary time is important to reduce health disparities among Aboriginal people. Therefore, the purpose of our study was 1) to determine the association between asthma and physical activity levels or sedentary time among Aboriginal adults overall, and among those of different Aboriginal identities, and 2) to understand the influence of physical inactivity and sedentary time on health care use among Aboriginal adults with asthma.

\section{Methods}

\section{Data and participants}

We used the 2006 Aboriginal Peoples Survey (APS) for the current analysis. The APS is a national survey of Aboriginal people in Canada administered by Statistics Canada. The objective of the APS is to examine issues such as education, language, employment, income, health, mobility and housing among Aboriginal people. The target population includes First Nations, Métis and Inuit people, 6 years and older, living off-reserve in rural, urban or northern areas. All APS data were selfreported through personal interviews conducted by phone or in person.

The 2006 APS sampled 61041 individuals, of which $80.1 \%$ responded. We used the public use microdata file for this study ( $\mathrm{n}=$ 24 368). Since this study focussed on adults, we excluded from our analysis 3415 individuals younger than 20 years $(n=20$ 953).

Detailed information about the APS sampling design, data collection and weighting can be found in the 2006 APS concepts and methods guide. ${ }^{20}$

\section{Main variables}

\section{Asthma}

APS participants were asked whether they had physician-diagnosed asthma. If they answered yes, they were asked whether they were currently receiving treatment or taking medication for their asthma. To ensure that participants had "current" asthma and to prevent misclassification associated with overdiagnosis, ${ }^{21}$ we classified those who responded in the affirmative to both questions as having asthma; all other respondents were considered not to have asthma. Defining asthma in this way led to the exclusion of 665 respondents.

\section{Insufficient physical activity (IPA)}

This variable was derived from the survey question: "In a typical week, how much time do you spend doing physical activities outside of work that result in an increase in your heart rate and breathing?' Response options were none; from 1 to 2 hours; from 3 to 4 hours; from 5 to 6 hours; from 7 to 10 hours; 11 hours or more. Current physical activity guidelines for adults (18-64 years) recommend at least 150 minutes of moderate- to vigorous-intensity aerobic activity per week; ${ }^{22}$ thus, we recoded responses into 2 categories: sufficiently physically active ( $\geq 3$ hours per week) and insufficiently physically active ( $<3$ hours per week). We chose this conservative threshold to minimize misclassification of physically inactive participants who over-report physical activity levels. There are no reliability or validity data available on this single item question from the APS; however, previous research has shown acceptable levels of validity (compared to data measured by an accelerometer) and reliability of recall of moderate- to vigorous-intensity physical activities. ${ }^{23,24}$

\section{Television-viewing time (TVT)}

This variable was derived from the survey question: "In a typical week in the past 3 months, how much time did you usually spend watching television?"' Response options were none; less than 1 hour; from 1 to 2 hours; from 3 to 5 hours; from 6 to 10 hours; from 11 to 14 hours; from 15 to 20 hours; 20 hours or more. Responses were recoded into 2 categories: high television-viewing time (TVT) ( $>10$ hours per week) and lower TVT ( $\leq 10$ hours per week). Current epidemiological research suggests that sedentary activity be limited to less than 2 hours per day or 14 hours per week, with standing breaks after $30 \mathrm{~min}$ utes of continuous sitting. ${ }^{25}$ TVT accounts for a large amount, but not all, of daily sitting time. ${ }^{17}$ Thus, our categorization is likely a conservative cut-point since adults also engage in other sedentary activities such as computer screen time and reading.

\section{Health care use}

The following 3 variables were used to assess health care use: spending 1 or more nights in the hospital in the past year (yes or no) or in the past 5 years (yes or no), and consulting a health care professional (including, but not limited to, family physicians, general practitioners, other medical doctors or specialists, nurses and First Nation, Métis or Inuit traditional healers) in the past 12 months (yes or no).

\section{Covariates}

One of the covariates in this study was Aboriginal identity, which included the response categories of "single identity: North American Indian only"; "single identity: Métis only"; "single identity: Inuk only”; "multiple identities”; “other Aboriginal identity”; "no Aboriginal identity”; and "non-Inuit in Arctic."

Individuals were selected for the APS based on 4 pre-screening questions in the Statistics Canada 2006 Census. These questions were designed to gather information about ethnic origin, Aboriginal selfreported identity (North American Indian, 
Métis, or Inuit), Indian band / First Nation membership and Treaty or Registered Indian status. Those who reported ancestral Aboriginal origin without an identity were considered part of the Aboriginal ancestryonly population and were included in the "no Aboriginal Identity" category.

Individuals with asthma in the "single identity: North American Indian only” (n = 715), "single identity: Métis only" ( $=598)$ and overall sample (all identities together; $\mathrm{n}=$ 1830) categories were further analyzed. The analysis of the overall sample provides useful information about all First Nations (offreserve), Métis, Inuit and other Aboriginal identities within Canada. We did not analyze other groups individually because of their limited sample size; these included single identity: Inuk only ( $\mathrm{n}=118$ ); multiple identities ( $\mathrm{n}=50$ ); other Aboriginal identity ( $\mathrm{n}=28$ ); no Aboriginal identity $(\mathrm{n}=314$ ) and non-Inuit in Arctic ( $\mathrm{n}=7$ ).

Other variables of interest included sociodemographic and health status. Survey respondents were provided with response options for these variables. Further details on the interpretation of these categories are available in the user guide. ${ }^{22}$

Previous research has indicated that age, sex and geographical location are covariates associated with asthma among all 4 adult Aboriginal groups, ${ }^{2}$ and that location has been linked to the availability of health care services and physical activity resources. $^{26}$ As a result, demographic covariates included sex (male or female), age categories $(20-24 ; 25-34 ; 35-44 ; 45-$ 54 ; or $\geq 55$ years) and geographical location (central metropolitan area; urban; rural or arctic).

Socioeconomic covariates included respondent education (elementary or less; some high school; completed high school; some post-secondary non-university; completed post-secondary non-university; some university; or completed university) and total household total income $(<\$ 20,000$; $\$ 20,000-39,999$; $\$ 40,000-59,999$; $\$ 60,000-$ 79,999; $\$ 80,000-99,999 ; \geq \$ 100,000)$. These covariates were included because socioeconomic factors have been linked to levels of health and fitness among Aboriginal adults. ${ }^{27}$
Smoking status (daily smoker; occasional smoker but former daily smoker; always an occasional smoker; non-smoker now, former daily smoker; non-smoker now, former occasional smoker; or never smoked) and self-reported body mass index (underweight [BMI < 18.5]; normal weight [18.5-24.9]; overweight [25.029.9]; obese Class I [30.0-34.9]; obese Class II [35.0-39.9]; or obese Class III [ $\geq$ 40.0]) were included because they are established health-risk factors. ${ }^{28}$

Covariate categories containing less than $5 \%$ of the sample were collapsed with an adjacent category. However, the underweight BMI category was not collapsed, as this is a distinct and important category of BMI.

\section{Statistical analysis}

Frequencies were used to describe the covariates and outcomes; these were converted into a percentage of the sample. Pearson chi-squares were used to determine whether there were differences between those with and without asthma. In cases where there were more than 2 categories, we used standardized adjusted residuals to determine where the difference was. A cutpoint of -2.0 to 2.0 was used to detect significance. Simple and covariate adjusted logistic regression analyses were used to assess the associations between asthma and IPA or TVT in each of the Aboriginal groups and in the overall sample comparing those with and without asthma. Logistic regressions were adjusted for age, sex, Aboriginal identity, geographical location, income, education, type of smoker and BMI covariates. In the sample of adults with asthma, simple and adjusted logistic regression models were used to assess the association between outcomes of health care use and IPA or TVT.

All statistical analyses were conducted using SPSS version 21 and significance was set at $\alpha<0.05$. In order to ensure appropriate estimations, normalized population weights provided by Statistics Canada were applied to the entire dataset.

\section{Results}

The prevalence of asthma in this sample was $8.7 \%$. Within the asthma group, $39.1 \%$ were North American Indian, 32.7\% were Métis, $11.1 \%$ were Inuit or non-Inuit in Arctic or other identity/multiple identities, and $17.2 \%$ were no Aboriginal identity. Table 1 shows characteristics of the sample. Significant differences were observed between those with treated asthma and those without asthma on most covariates examined. The proportion of those who were insufficiently physically active was higher among those with asthma (56.9\%) compared to those without asthma $(50.9 \%)$. The proportion of those who reported high TVT was higher among those with asthma (50.4\%) compared to those with no asthma (42.9\%).

Adults with asthma were significantly more likely to report high TVT and IPA compared to those without asthma in unadjusted models (Table 2). This was true for the North American Indian, Métis and the overall samples. These associations remained significant after adjusting for age, sex, Aboriginal identity, geographical location, income, education, type of smoker and BMI.

The odds of health care use were higher among adults with asthma who had high TVT in unadjusted and adjusted models (Table 3). For IPA, all unadjusted associations with health care use were significant. For health care professional consultations in the unadjusted regression, the association was negative such that IPA was associated with fewer consultations $(\mathrm{OR}=0.77$; CI: 0.71-0.84), while the association between IPA and hospital night stays in the past year $(\mathrm{OR}=1.20$; CI: $1.13-1.27)$ and past 5 years $(\mathrm{OR}=1.49$; CI: 1.43-1.56) were both positive. In models adjusted for all covariates, hospital night stays in the past year and 5 years were both significantly associated with IPA.

\section{Discussion}

Using a sample of adults from the 2006 APS, we analyzed the relationships between physical inactivity and TVT with asthma and health care use. The primary finding is that adults with asthma were significantly more likely to be in the high televisionviewing-time group ( $>10$ hours per week) and to be insufficiently physically active ( $<3$ hours per week) compared to those 
TABLE 1

Characteristics of the sample by self-reported asthma, Aboriginal Peoples Survey, 2006

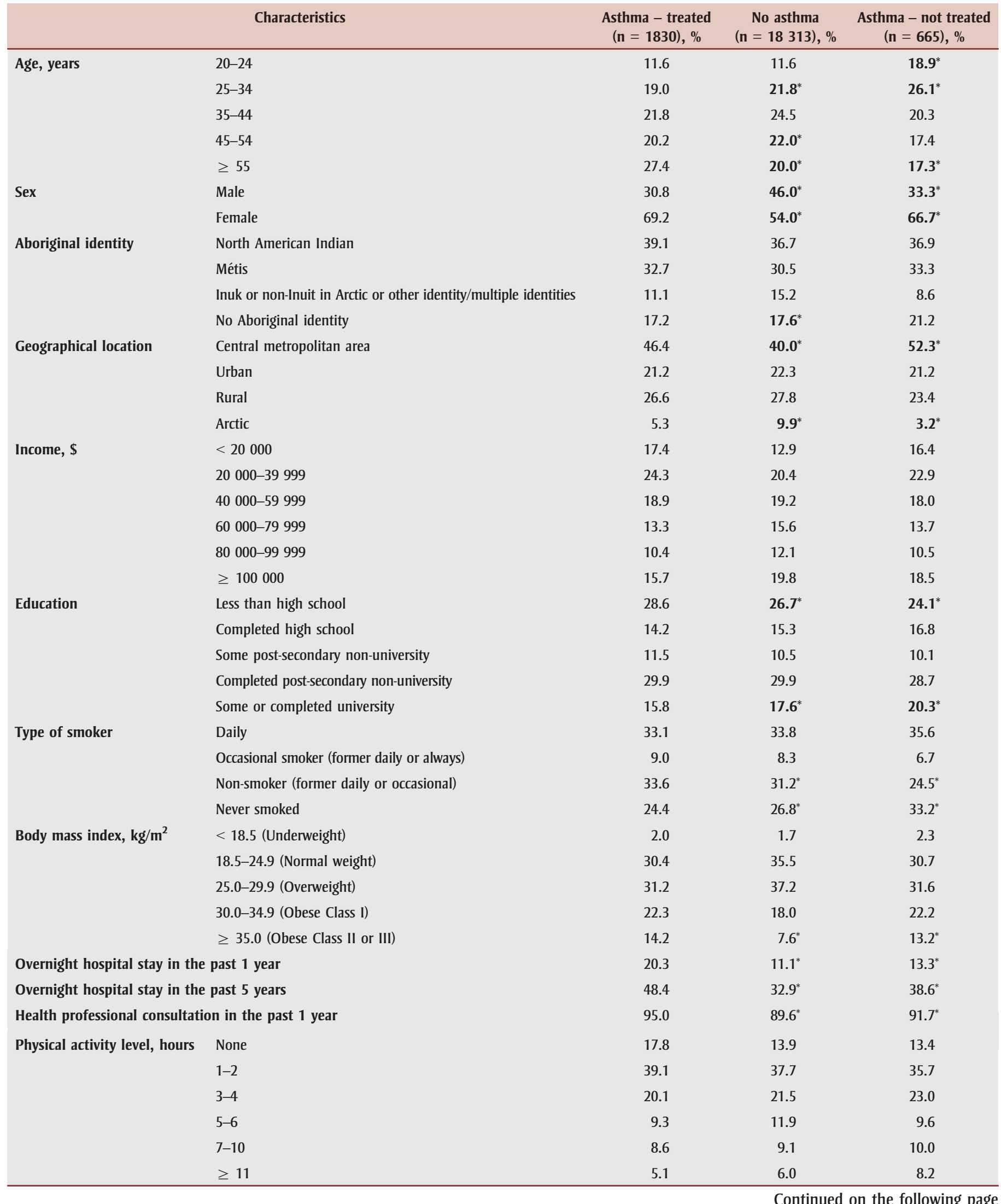

Continued on the following page 
TABLE 1 (continued)

Characteristics of the sample by self-reported asthma, Aboriginal Peoples Survey, 2006

\begin{tabular}{|c|c|c|c|c|}
\hline & Characteristics & $\begin{array}{l}\text { Asthma - treated } \\
(n=1830), \%\end{array}$ & $\begin{array}{c}\text { No asthma } \\
(\mathrm{n}=18313), \%\end{array}$ & $\begin{array}{l}\text { Asthma - not treated } \\
\qquad(\mathrm{n}=665), \%\end{array}$ \\
\hline Physical activity & Insufficient (< 3 hours/week) & 56.9 & $50.9^{*}$ & $54.8^{*}$ \\
\hline Television time & Sedentary (> 10 hours/week) & 50.4 & $42.9^{*}$ & $42.6^{*}$ \\
\hline
\end{tabular}

${ }^{*} p<.05$.

without asthma; this was true for the North American Indian and Metis groups as well as the overall sample. The secondary finding is that adults with asthma who reported high TVT used health care services more than those who reported lower TVT, and that adults with asthma who were insufficiently physically active reported more hospital stays than those who were sufficiently active. While previous research has concluded that individuals with asthma are more likely to report low physical activity levels ${ }^{29}$ and higher health care utilization, ${ }^{12}$ this is the first study to investigate these relationships within the Aboriginal population in Canada and within different Aboriginal identities, each of which may be at varying risk due to a multitude of health disparities. ${ }^{30}$
Our finding that Aboriginal adults with asthma are more likely to report lower levels of physical activity is consistent with findings from research on nonAboriginal adults with asthma. ${ }^{28}$ Many individuals with asthma avoid physical activity for fear of exacerbating symptoms or triggering exercise-induced bronchoconstriction. ${ }^{31}$ However, numerous studies have shown that physical activity can improve asthma control and asthmarelated quality of life. ${ }^{11,31}$ Dogra et al. ${ }^{12}$ investigated the relationship between physical activity and health in 4272 men and 6971 women with asthma from the Canadian Community Health Survey cycle 2.1. Data showed that physical activity was consistently associated with better health in individuals with asthma. ${ }^{12}$
Further, engaging in regular, structured physical activity has been shown to improve asthma control in adults with asthma. ${ }^{11}$ Thus, physical activity promotion strategies among Aboriginal people may benefit from an asthma education component, and asthma education should include information on physical activity.

The results of our study also indicate that North American Indians living off-reserve, Métis and the overall Aboriginal population group with asthma were more likely to report high TVT compared with those without asthma. Only a limited number of studies exist on the association between asthma and TVT, and these are primarily on children. In one such study, children presenting with no wheeze at 3.5 years

TABLE 2

Television-viewing time and physical inactivity in North American Indian populations, Métis populations and the overall Aboriginal sample, $\geq 20$ years, Aboriginal Peoples Survey, 2006

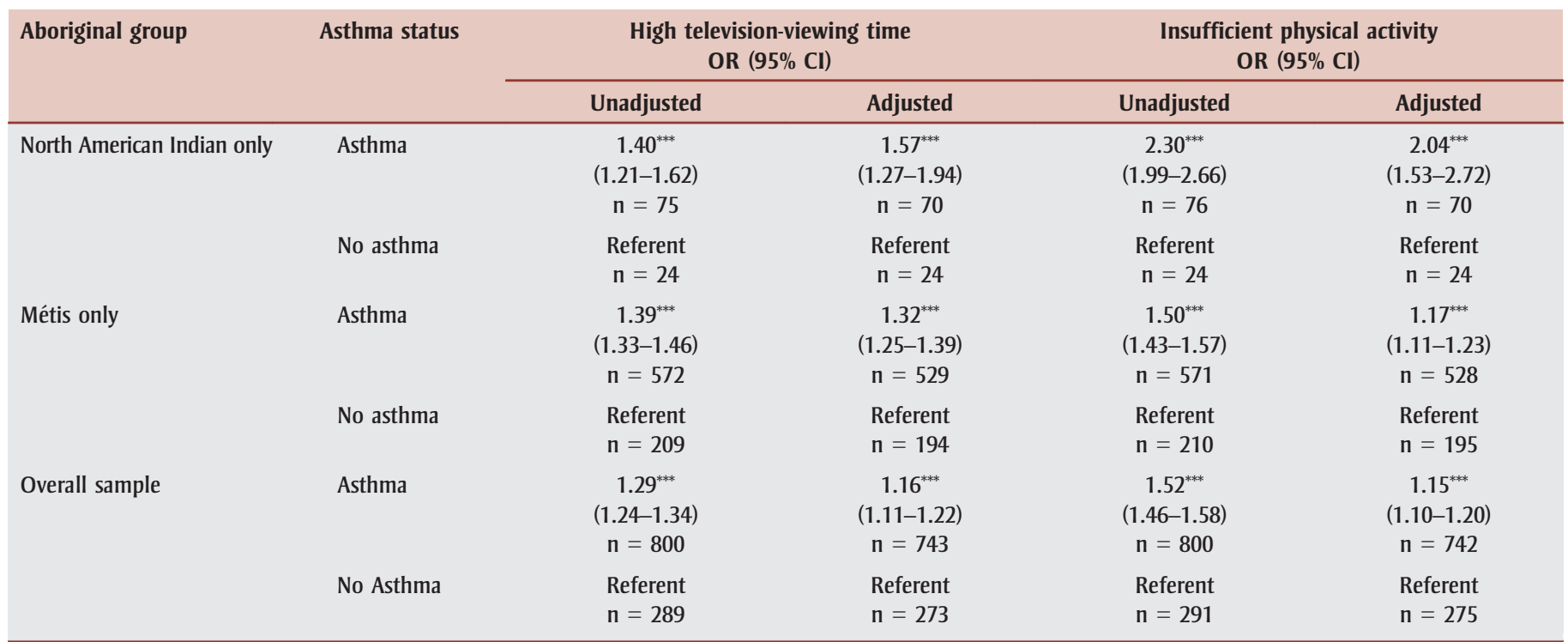

Abbreviations: BMI, body mass index; $\mathrm{CI}$, confidence interval; OR, odds ratio.

Notes: Response rates for physical activity and television-viewing time variables were $8.2 \%$ in North American Indians compared to $95 \%$ in Métis.

Adjusted for age, sex, geographical location, income, education, type of smoker, and BMI.

*** $p<.001$. 
TABLE 3

Association between health care utilization and physical inactivity or television-viewing time among Aboriginal adults ( $\geq 20$ years) with asthma, Aboriginal Peoples Survey, 2006

\begin{tabular}{|c|c|c|c|c|c|c|c|}
\hline \multicolumn{2}{|c|}{ Activity type } & \multicolumn{2}{|c|}{$\begin{array}{l}\text { Health care professional } \\
\text { consultation, } \\
\text { OR }(95 \% \mathrm{CI})\end{array}$} & \multicolumn{2}{|c|}{$\begin{array}{c}\text { Overnight hospital stay } \\
\text { in the past year, } \\
\text { OR }(95 \% \mathrm{Cl})\end{array}$} & \multicolumn{2}{|c|}{$\begin{array}{c}\text { Overnight hospital stay } \\
\text { in the past } 5 \text { years, } \\
\text { OR }(95 \% \mathrm{Cl})\end{array}$} \\
\hline & & Unadjusted & Adjusted $^{\mathbf{b}}$ & Unadjusted & Adjusted $^{\mathbf{b}}$ & Unadjusted & Adjusted $^{\mathbf{b}}$ \\
\hline \multirow[t]{2}{*}{$\begin{array}{l}\text { Television screen time } \\
>10 \text { hours/ week }\end{array}$} & Sedentary & $\begin{array}{c}2.41^{* * *} \\
(2.21-2.63) \\
n=403\end{array}$ & $\begin{array}{c}2.59^{* * *} \\
(2.34-2.87) \\
n=372\end{array}$ & $\begin{array}{c}2.19^{* * *} \\
(2.07-2.32) \\
n=401\end{array}$ & $\begin{array}{c}1.95^{* * *} \\
(1.82-2.08) \\
n=370\end{array}$ & $\begin{array}{c}1.47^{* * *} \\
(1.41-1.53) \\
n=396\end{array}$ & $\begin{array}{c}1.13^{* * *} \\
(1.07-1.18) \\
n=370\end{array}$ \\
\hline & Active & $\begin{array}{l}\text { Referent } \\
n=397\end{array}$ & $\begin{array}{l}\text { Referent } \\
n=371\end{array}$ & $\begin{array}{l}\text { Referent } \\
n=394\end{array}$ & $\begin{array}{l}\text { Referent } \\
n=369\end{array}$ & $\begin{array}{l}\text { Referent } \\
n=401\end{array}$ & $\begin{array}{l}\text { Referent } \\
n=370\end{array}$ \\
\hline \multirow[t]{2}{*}{$\begin{array}{l}\text { Insufficient physical } \\
\text { activity }<3 \text { hours/week }\end{array}$} & Insufficiently active & $\begin{array}{c}0.77^{* * *} \\
(0.71-0.84) \\
n=455\end{array}$ & $\begin{array}{c}0.98 \\
(0.89-1.08) \\
n=422\end{array}$ & $\begin{array}{c}1.20^{* * *} \\
(1.13-1.27) \\
n=453\end{array}$ & $\begin{array}{c}1.16^{* * *} \\
(1.08-1.23) \\
n=421\end{array}$ & $\begin{array}{c}1.49^{* * *} \\
(1.43-1.56) \\
n=452\end{array}$ & $\begin{array}{c}1.22^{* *} \\
(1.16-1.28) \\
n=419\end{array}$ \\
\hline & Active & $\begin{array}{l}\text { Referent } \\
n=345\end{array}$ & $\begin{array}{l}\text { Referent } \\
n=320\end{array}$ & $\begin{array}{l}\text { Referent } \\
n=342\end{array}$ & $\begin{array}{l}\text { Referent } \\
n=317\end{array}$ & $\begin{array}{l}\text { Referent } \\
n=345\end{array}$ & $\begin{array}{l}\text { Referent } \\
n=320\end{array}$ \\
\hline
\end{tabular}

Abbreviations: $\mathrm{Cl}$, confidence interval; OR, odds ratio.

a Including, but not limited to, family physicians, general practitioner, other medical doctor or specialist, nurse or First Nation, Métis or Inuit traditional healer.

b Adjusted for age, sex, Aboriginal identity, geographical location, income, education, type of smoker and body mass index.

** $p<.01$.

${ }^{* * *} p<.001$.

were followed over a 7.5-year prospective longitudinal study, during which television screen time rates were recorded. ${ }^{32}$ The results showed that children who watched an average of 2 or more hours of television per day over the 7.5-year period were more than twice as likely to develop asthma by 11.5 years of age. ${ }^{32}$ Further, a number of adverse health outcomes, including obesity, behavioural problems and decreased academic performance, are associated with excessive screen time ${ }^{33}$ and are all more prevalent among children with asthma. ${ }^{34}$ Recent evidence has also indicated that sedentary behaviour, including TVT, is an important risk factor for cardiovascular disease and all-cause mortality independent of physical inactivity. ${ }^{35}$ Since Aboriginal people are already at a higher risk of many of these adverse health outcomes, ${ }^{1}$ the high TVT reported among Aboriginal adults with asthma in this study highlights a potential target for future health promotion strategies.

Research has shown that Aboriginal people in Canada visit the hospital more than twice as frequently as do their nonAboriginal peers. ${ }^{3}$ Our study found that adults with asthma who reported high TVT had higher health care utilization. This is not surprising given the multitude of adverse health outcomes associated with high TVT. ${ }^{31-33}$ Similarly, adults with asthma who were insufficiently active had significantly higher odds of being hospitalized overnight in the past year. This is consistent with previous results from the Canadian Community Health Survey that indicated that physically active adults with asthma use health care services less than inactive adults with asthma do; that is, they have better asthma control. ${ }^{12}$ Of note, the association between physical inactivity and health care use was less consistent; no statistical significance was noted for health care professional consultations. The inconsistent findings for physical inactivity and health care utilization, especially consultations with a professional, may be confounded by exerciseinduced asthma, that is, active adults with asthma may be seeking health care services to ensure they have medications to prevent inactivity related to asthma symptoms. Future research should consider more objective measures of asthma control and should consider exercise-induced asthma.

Evidence is growing that the impact of screen time on health outcomes is independent of physical activity; ${ }^{35}$ from this preliminary descriptive study, it is difficult to determine whether sedentary time may be more important than physical inactivity for asthma outcomes in this population. Certainly, more research is required. Regardless, data from the present study clearly show that Aboriginal adults with asthma need to increase their physical activity and reduce their TVT; doing so could lead to an increase in asthma control, a decrease in health care costs and likely better quality of life.

\section{Strengths and limitations}

The strengths of this analysis include the large sample size, which allowed for analysis in 2 distinct Aboriginal identities, and the number of available variables in the APS that were adjusted for in regression models. Results of this study should be interpreted in light of the following limitations.

First, the data used in this study were cross-sectional; thus, reverse causality cannot be ruled out. Second, covariates not measured in the study, such as exercise-induced asthma, could be responsible for a confounding variable effect. Third, all data were self-reported, which may have led to misclassification, especially in regards to physical activity, sedentary behaviour and BMI. For physical activity and sedentary behaviour, 
recall has been shown to have acceptable validity and reliability when using 7-day recall questionnaires ${ }^{23,24}$ whereas selfreported BMI can accurately predict measured BMI. ${ }^{36}$ However, objectively measured data are better able to predict health outcomes $^{37}$ when compared to self-reported data. As such, conservative cut-points were deliberately chosen to minimize bias in the present study. Future research should use objectively measured or valid/reliable tools to measure these variables.

Fourth, the sample did not include onreserve First Nations and the sample size was not large enough to run individual analyses for all Aboriginal identities, both of which may limit the generalizability of the findings. It should also be noted that the APS does not contain information necessary for detailed analyses of health care accessibility within these identities. Future research should assess asthma among First Nations, Métis and Inuit separately to better understand the associations within these specific populations. Fifth, the APS did not contain information pertaining to asthma control, so health care use outcomes were used as a proxy. Future research should directly assess the association between IPA, TVT and asthma control in Aboriginal adults with asthma, both on- and off-reserve. Finally, the present analysis was the first to explore such associations in Aboriginal adults. Future research should test for potential effect modifiers to determine if the associations differ by variables such as smoking status, medication use and others.

\section{Conclusion}

Data from the APS indicate that Aboriginal adults with asthma are more sedentary and less physically active than their non-asthmatic peers. Further, Aboriginal adults with asthma who report higher levels of TVT are more likely to use health care services than their less sedentary peers. These novel findings have important implications for asthma-exercise education in the Aboriginal population in Canada.

\section{Conflict of interest}

The authors report no conflicts of interest. The authors alone are responsible for the content and writing of the paper.

\section{References}

1. King M. Chronic diseases and mortality in Canadian Aboriginal peoples: learning from the knowledge. Chronic Dis Can. 2010;31(1): 2-3.

2. Change HJ, Beach J, Senthilselvan A. Prevalence and risk factors of asthma in off-reserve Aboriginal children and adults in Canada. Can Respir J. 2012;16(6):e68-74.

3. Crighton E, Wilson K, Senecal S. The relationship between socio-economic and geographic factors and asthma among Canada's Aboriginal populations. Int J Circumpolar Health. 2010;69(2):138-50.

4. Chen Y, Johansen H, Thillaiampalam S, Sambell C. Asthma. Health Reports. 2005; 16(2):43-6.

5. Wilson C. The Canadian Lung Association/ L'association pulmonaire du Canada to PreBudget Consultations House of Commons Standing Committee on Finance. Ottawa (ON): The Lung Association; 2011.

6. Senthilselvan A, Habbick BF. Increased asthma hospitalizations among registered Indian children and adults in Saskatchewan, 1970-1989. J Clin Epidemiol. 1995;48(10): 1277-83.

7. Warburton DE, Katzmarzyk PT, Rhodes RE, Shephard RJ. Evidence-informed physical activity guidelines for Canadian adults. Can J Public Health. 2007;32(S2E):S16-68.

8. Humphreys BR, McLeod L, Ruseski JE. Physical activity and health outcomes: evidence from Canada. Health Econ. 2014;23(1): 35-54.

9. Young TK, Katzmarzyk PT. Physical activity of Aboriginal people in Canada. Appl Physiol Nutr Metab. 2007;32 Suppl 2:S14860 .

10. Statistics Canada. Physical activity during leisure time, 2011 [Internet]. Ottawa (ON): Statistics Canada; [cited 2013 Nov 21]. Available from: http://www.statcan.gc.ca /pub/82-625-x/2012001/article/11667-eng .htm

11. Dogra S, Kuk JL, Baker J, Jamnik V. Exercise is associated with improved asthma control in adults. Eur Respir J. 2011;37:318-23.
12. Dogra S, Baker J, Ardern CI. The role of physical activity and body mass index in the use of adults with asthma. Ann Allergy Asthma Immunol. 2009;102(6):462-8.

13. Cochrane LM, Clark CJ. Benefits and problems of a physical training programme for asthmatic patients. Thorax. 1990;45(5):34551.

14. Owen N, Healy GN, Matthews CE, Dunstan DW. Too much sitting: the populationhealth science of sedentary behaviour. Exerc Sport Sci Rev. 2010;38(3):105-13.

15. Thorp A, Owen N, Neuhaus M, Dunstan D. Sedentary behaviors and subsequent health outcomes in adults a systematic review of longitudinal studies, 1996-2011. Am J Prev Med. 2011;41(2):207-15.

16. Tremblay MS, Colley RC, Saunders TJ, Healy GN, Own N. Physiological and health implications of a sedentary lifestyle. Appl Physiol Nutr Metab. 2010:35(6):725-40.

17. Shields M, Tremblay M. Sedentary behaviour and obesity. Health Rep. 2008;19(2):19-30.

18. Janz T, Seto J, Turner A. Aboriginal Peoples Survey, 2006: an overview of the health of the Métis population. Ottawa (ON): Statistics Canada, Social and Aboriginal Statistics Division; 2009. [Statistics Canada, Catalogue No.: 89-637-X - No. 004.]

19. Sin DD, Wells H, Svenson LW, Man SF. Asthma and COPD among aboriginals in Alberta, Canada. Chest. 2002;121(6):1841-6.

20. Statistics Canada. Aboriginal Peoples Survey, 2006: concepts and methods guide. Ottawa (ON): Statistics Canada, Social and Aboriginal Statistics Division; 2009. [Statistics Canada, Catalogue No.: 89-637-X - No. 003.]

21. Aaron SD, Vandemheen KL, Boulet LP, et al. Overdiagnosis of asthma in obese and nonobese adults. CMAJ. 2008;179(11):1121-31.

22. Canadian Society for Exercise Physiology. Canadian physical activity guidelines [Internet]. Ottawa (ON): CSEP (2011); [cited 2013 Nov]. Available from: http://www.csep .ca/CMFiles/Guidelines/CSEP_PAGuidelines_ adults_en.pdf

23. Craig CL, Marshall AL, Sjostrom M, et al. International physical activity questionnaire: 12-country reliability and validity. Med Sci Sports Exerc. 2003;35(8):1381-95. 
24. Zuazagoitia A, Montoya I, Grandes G, et al. Reliability and validity of the 7-day Physical Activity Recall interview in a Spanish population. Eur J Sport Sci. 2014;14(1): S361-8.

25. Owen N, Sugiyama T, Eakin EE, Gardiner PA, Tremblay MS, Sallis JF. Adults' sedentary behavior determinants and interventions. Am J Prev Med. 2011;41(2):189-96.

26. Sibley LM, Weiner JP. An evaluation of access to health care services along the rural-urban continuum in Canada. BMC Health Serv Res. 2011;11(20):1-11.

27. Garner R, Carrière G, Sanmartin C; Longitudinal Health and Administrative Data Research Team. The health of First Nations living off-reserve, Inuit, and Métis adults in Canada: the impact of socioeconomic status on inequalities in health. Ottawa (ON): Statistics Canada, Health Information and Research Division; 2010. [Statistics Canada, Catalogue No.: 82-622-X - No. 004.]

28. Koster A, Leitzmann MF, Schatzkin A, et al. The combined relations of adiposity and smoking on mortality. Am J Clin Nutr. 2008;88(5):1206-12.

29. Ford ES, Heath GW, Mannino DM, Redd SC. Leisure-time physical activity patterns among US adults with asthma. Chest. 2003;124(2):432-7.

30. Lix LM, Bruce S, Sarkar J, Young TK. Risk factors and chronic conditions among Aboriginal and non-Aboriginal populations. Health Rep. 2009;20(4):21-9.

31. Mancuso C, Sayles W, Robbins L, et al. Barriers and facilitators to healthy physical activity in asthma patients. $\mathrm{J}$ Asthma. 2006;43(2):137-43.

32. Sherriff A, Maitra A, Ness AR, Mattocks C, Riddoch C, Reilly JJ. Association of duration of television viewing in early childhood with the subsequent development of asthma. Thorax. 2009;64(4):321-5.

33. American Academy of Pediatrics: Committee on Public Education. American Academy of Pediatrics: children, adolescents, and television. Pediatrics. 2001;107(2):423-6.
34. Conn KM, Hernandez T, Puthoor P, Fagnano M, Halterman JS. Screen time use among urban children with asthma. Acad Pediatr. 2009;9(1):60-3.

35. Stamatakis E, Hamer M., Dunstan D. Screen-based entertainment time, all-cause mortality, and cardiovascular events: population-based study with ongoing mortality and hospital events follow-up. J Am Coll Cardiol. 2011;57(3):292-9.

36. Scribani M, Shelton J, Chapel D, et al. Comparison of bias resulting from two methods of self-reporting height and weight: a validation study. JRSM Open. 2014;5(6): 2042533313514048.

37. Shephard RJ. Objective vs. self-reported physical activity and sedentary time: effects of measurement method on relationships with risk biomarkers. Br J Sports Med. 2003;37:197-206. 